Case Report

\title{
Munchausen's syndrome in plastic surgery practice: A bewildering situation!
}

\author{
D. Nardella, M. S. Sohawon, O. Heymans \\ Department of Plastic Surgery, Liège, Belgium
}

Address for correspondence: Dr. Daniele Nardella, Department of Plastic Surgery, CHU Sart Tilman, University of Liege, Belgium. E-mail: nardellad@hotmail.com

\begin{abstract}
The plastic surgeon rarely encounters patients expressing factitious disorders. Through their incredible imagination, these patients conjure numerous lesions for themselves and willingly accept to undergo invasive diagnostic procedures and risky therapies. We report four cases of Munchausen's syndrome in the field of plastic surgery and follow with a discussion of as to when should the alarm bells start ringing for the unsuspecting plastic surgeon, to assist him in dealing with these too often-ignored disorders.
\end{abstract}

\section{KEY WORDS}

Factitious disorders, Munchausen's syndrome, plastic surgery

atients having ulcers and chronic wounds from different origins often seek medical advice from plastic surgeons. Although these different cutaneous wounds often have a traumatic origin, we frequently encounter ulcers resulting from malignancy, infections or autoimmune origin. Faced with a nontraumatic wound or ulcer, the history and clinical examination generally allows the surgeons to make the correct diagnosis and give the appropriate treatment. However, plastic surgeons are often consulted for lesions for which the origin is unclear despite multiple investigations and unsuccessful attempts at treatment. It is very difficult then for the $\mathrm{n}^{\text {th }}$ consulted doctor who must start the process anew. Additionally, diagnoses for such patients are frequently inconclusive and conflicting. In this context, factitious disorders have to be considered. After studying four such cases, we propose an approach in order to achieve a correct diagnosis and management programme.

\section{CASE REPORTS}

In the past three years, 8000 patients underwent surgery while 30,000 patients were taken care of in the outpatient clinic of our department in the University Hospital in Liege. This clinical activity involved fields such as aesthetic, maxillo-facial, hand, general plastic surgery, microsurgery, free tissue transfer and burn care. Although primary wound care is part of the outpatient clinic activity, it rarely presents a problem. However, we classified four especially difficult cases as Munchausen's syndrome, each having its own highly specific attributes.

\section{Case 1}

A 54-year-old female nurse consulted us for two ulcers on her forehead without any previous trauma history [Figure 1]. These superficial, painless and isolated wounds seemed to have been evolved for a few weeks. Since there was no obvious diagnosis, the lesions were 
given local treatment. Despite this simple approach, the lesions worsened. This evolution seemed atypical, so we proposed a biopsy. The first biopsy only showed the existence of a granulomatous tissue of unclear origin. Later another ulcer appeared, but the patient treated it by herself. She described an alternation between the closing and opening of the different wounds over a few months' time, but she was not able to understand the dynamics of the phenomenon. We then lost track of the patient. Later, a biopsy was taken elsewhere in another institution due to aggravation of her ulcer, this evoked signs of vasculitis. The diagnosis of Kimura's disease was presented as a possibility. Local care and chemotherapy based on methotrexate were prescribed for a nine-month period with limited success.

Seven years later, after having consulted many doctors from different institutions, this patient returned for

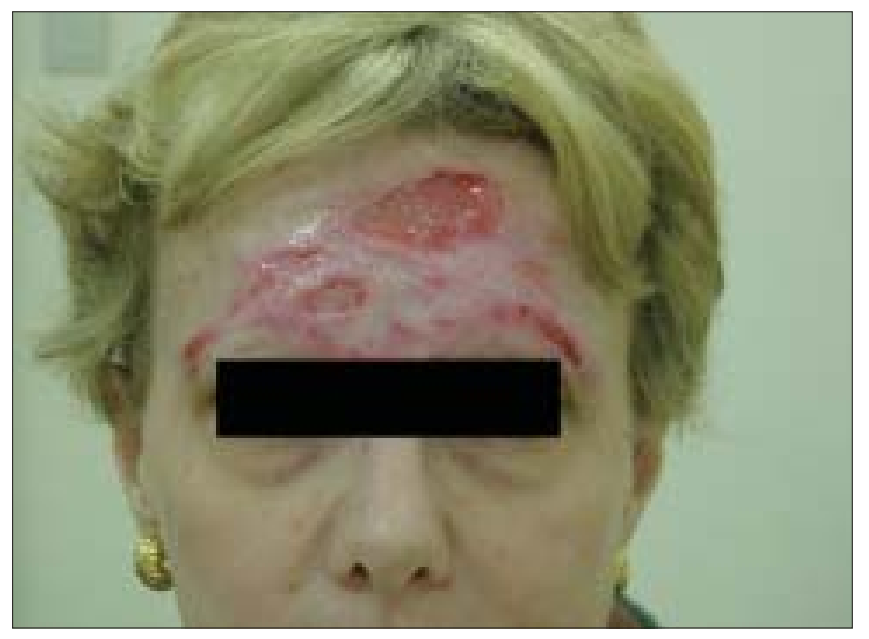

Figure 1: Case report 1

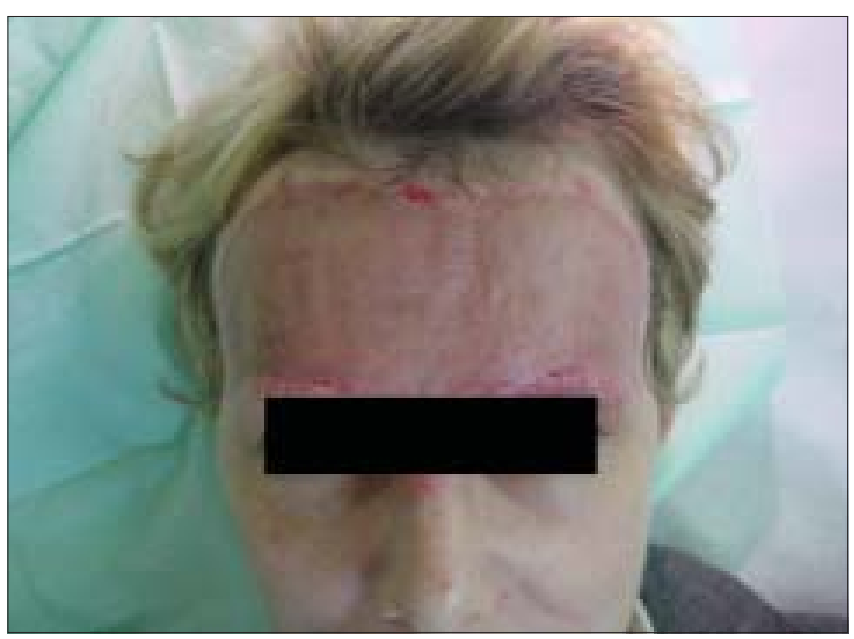

Figure 2: Case report 1 after skin graft of the forehead the same problem. Confronted with all this uncertainty and the large ulcer surrounded by a zone of scarring on the forehead (35 mm) [Figure 1], we decided to make another resection of the scar tissue. After excision of the forehead region, a skin graft was done [Figure 2]. Although this biopsy didn't help in its aetiology, the pathologist concluded that there was an epithelial lesion with a pattern of "scraping" around the wound. We clearly saw discordance between the reality of the lesions and the absence of a definitive diagnosis, especially without trauma. In spite of the seemingly accurate diagnosis of Kimura's disease, the therapeutic dilemma was obvious. The scraping pattern, attested by histological examination, left us puzzled. We concluded that the correct diagnosis should be selfmutilation. After many discussions with the patient, she finally revealed the truth. A difficult family situation was contemporary with the development of these lesions: divorce, loss of her parental rights. Her profile corresponded with the stereotypical profile for this kind of fictitious disease: nurse, wealthy socioeconomic class, depression.

\section{Case 2}

A 26-year-old man, who was overweight, consulted us for a wound dehiscence located on a laparotomy scar. The medical history of this patient was considerable. The surgical history began three years ago with a gastric banding. The postoperative period seemed to have been difficult with fistula and abdominal wall infection. After a new operation, the gastric fistula was controlled but a second abdominal wall infection began. The patient described his journey through different surgeons and

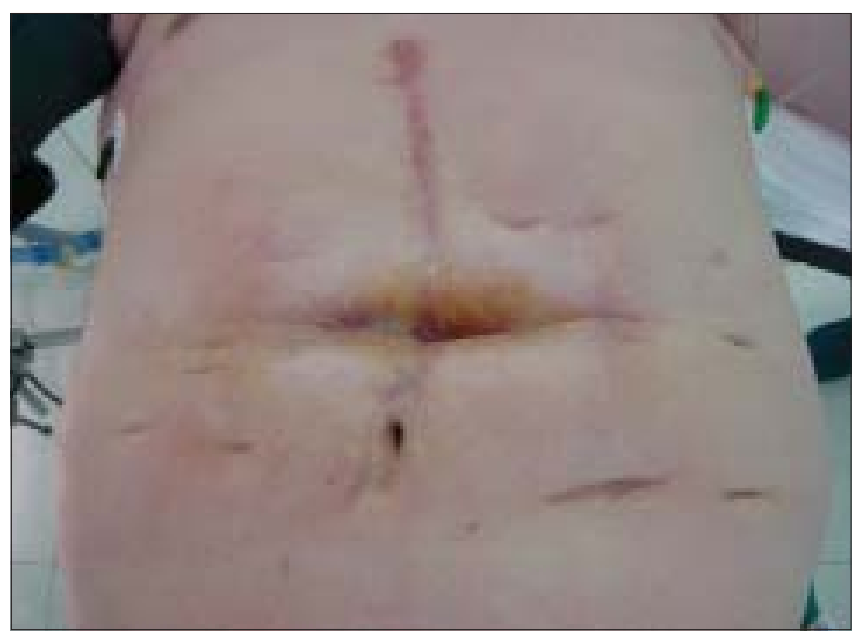

Figure 3: Case report 2 
institutions. Diagnoses ranged from Crohn's ileitis to recurrence of fistula. Multiple bacterial cultures showed Pseudomonas aeruginosa and Staphylococcus aureus. The patient reported about 33 surgical interventions and there was still a cicatricial defect.

At this point, the patient had a weight loss of about $10 \mathrm{~kg}$. The laparotomy scar was $5 \mathrm{~cm}$ long, being totally umbilicated [Figure 3]. The deep part of this wound was moist, discharging and foul-smelling. The CT abdominal scan showed an inflammatory process surrounding this invaginated zone, without sign of digestive fistula. Due to the soft tissue redundancy linked to the patient's obesity, we decided, to perform a large resection of the pathological tissues. After the resection, we proceeded to a direct closure, without tension.

Three days after the operation, the dressings were removed, the wound was opened over $5 \mathrm{~cm}$, showing $6 \mathrm{~cm}$-thick subcutaneous fat. The non-absorbable thread had disappeared. We decided to proceed to the closure of this dehiscence for a second time. Five days later, the wound was opened again.

As in Case Report 1, there was still discordance between the reality of the lesions and the absence of explanation. The 33 surgical interventions in different institutions were of great concern. The complete disappearance of the non-abso rbable threads three days after the operation suggested auto-mutilation. This suspicion was confirmed by the second intervention. A frank discussion with the patient and evaluation by a psychiatrist confirmed our diagnosis. This discussion revealed a troubled family background and a refusal of the gastric banding. The patient told us that his overweight status "wasn't a problem" and that he had "some regrets" regarding his surgery. A new wound closure, followed by a strictly closed dressing, finally led to healing.

\section{Case 3}

A 28-year-old man requested treatment for a painful ulcerated lesion located on the buccal side of the right comissure. This lesion measured $3 \mathrm{~cm}$ in diameter, but was very deep. No mucosa remained and the musculature was partially destroyed. Nevertheless, the wound was clean. Quickly and without specific reasons, the patient revealed the self-inflicting origin of the problem. Local treatment and analgesics were prescribed but the patient was lost on follow-up.

\section{Case 4}

A 23-year-old female veterinary student was examined for a red swelling of her left thigh. This problem was combined with fever. Medical workup showed a major inflammatory syndrome due to a deep, intramuscular abscess. Surgical drainage and debridement were performed. Bacteriological samples taken during the operation showed the presence of Enterococcus faecalis and Streptococcus feacium. We managed the wound until closure without problem. However, three weeks later, the patient presented again with the scar partially opened. She reported a spontaneous opening of the wound with simultaneous pus drainage. Because of the previous infectious origin, we had no doubt about her problem. After a short local treatment, the wound closed again.

Two months after the first episode and a perfect evolution, a tumefaction reoccurred on the same site showing redness, firmness and pain. Ultrasonography demonstrated an abscess. We drained the abscess, debrided and cleaned the wound once again.

Finally, some weeks later, she presented again with a marked redness on her thigh. We shared our suspicions of self-inflicted lesions with her mother. Shortly after our diagnosis, which appeared correct, the patient was lost on follow-up.

\section{DISCUSSION}

These four cases just described are factitious disorders, as defined by the DSM IV [Table 1]. ${ }^{[1]}$ This nosologic group contains different entities, the Munchausen syndrome representing $10 \%$ of it. ${ }^{[2]}$ These presentations differ from simulation in which the illness is induced or enhanced in order to obtain external incentives (often material). Disorders in which symptoms are the result of unconscious defence mechanisms, ${ }^{[1]}$ explain the Munchausen syndrome.

The plastic surgeon's daily practice is confronted with cutaneous injuries of different origins, viz. a possible

Table 1: Definition of the factitious disorders from the DSM

A. Production or intentional feint of either physical or psychological symptoms

B. The behaviour's motivation is to play the ill's role

C. Absence of external reasons for this behaviour (for example: to get money, to flee a legal responsibility or to better the material or physical situation as in the simulation) 
abuse of solar exposure, symptoms related to arterial and/or venous deficiency or of autoimmune aetiology. Moreover, the location and aspect of the injury are often evocative. Hence, the self-inflicting diagnosis is an exclusion diagnosis. Indeed, the surgeon rarely thinks that the patient could be the real cause of the non-healing of his wound. It is also difficult to arrive at an accurate diagnosis when the patient does not collaborate with the surgeon but is against him, even though unconsciously. Hamolsky is not reluctant to qualify these patients as adverse. ${ }^{[3]}$

The alarm bells should start ringing for the unsuspecting plastic surgeon when there exists an incoherence between the lesion's appearance, location, it's histopathological findings and its natural evolution. Especially, the time it takes to heal or not. The long medical history of multiple medical advices from many different physicians, from different hospitals, with frequent hospitalisations that are generally abnormally long, is also to be taken into consideration [Table 2]. Usually, the patient is a woman (sex ratio $20: 1)^{[4,5]}$ coming from a favourable socioeconomic background, often in close contact with the medical field, enhancing the construction of a plausible clinical board. ${ }^{[6]}$ This unconscious fantasy is often initiated through a previous illness or owing to familial or professional reasons. Furthermore, there exists an underlying personality disorder in these patients, particularly the borderline type [Table 3] together with a certain degree of unconscious sado-masochistic behaviour.

Traditionally, the tendency is to make a positive diagnosis, considering what the patient tells the surgeon is reliable and trustworthy. But when the above-named features are considered, although disturbing and generally disruptive to the patient's management, an accurate diagnosis of factitious disorder is possible.

\section{Table 2: Key elements in Munchausen's syndrome}

1. Unexplained natural history of wound healing
2. Long medical history, with numerous operations
3. Personality disorder of the patient
4. Female, paramedical profession, favourable socioeconomic
background
5. Bewildering situation faced by the surgeon
6. Patient usually lost on follow-up once unmasked

\section{Table 3: Essential borderline personality traits}

1. Little control over impulses

2. Unstable self-image and emotions

3. Unstable personal relationships
The patient with a borderline personality expresses an unconscious sadistic behaviour towards the surgeon, who will "suffer" from not being able to find an organic cause to the complaints of his patient. This sadistic relationship is gratifying to the patient and it encourages him/her to go and consult another surgeon afterwards. The masochistic side implies that the patient is willing to accept and undergo invasive diagnostic procedures numerous times, which can be qualified as an "acceptance of physical torture".

While under hospital care, they improve their medical knowledge through conversations with other patients and the medical staff. ${ }^{[7]}$ Medical textbooks and websites are also useful references for these "actor-patients". Nevertheless, this behaviour contrasts with the little details that they are able to give concerning the development of their lesions. ${ }^{[8]}$ Finally, they are often transferred from one specialised unit to the next, within university hospitals, with the suspicion of a rare illness diagnosis.

Self-inflicted wounds are probably the main aspect in factitious disorders in the surgical field. The problems are of three types: bad healing, recurrent infection and insertion of a foreign body. ${ }^{[4]}$ Some cases are more deceitful: for instance, superficial abscesses induced by salivary injections. In the immediate postoperative period, the healing deteriorations appear as superficial infections. Despite a surgical incision and an antimicrobial therapy, the infectious phenomenon recurs. ${ }^{[5]}$ In aesthetic surgery, diagnosing the disorder is difficult since the interventions are often made according to the patient's wishes. ${ }^{[9]}$

Although, the factitious disorder diagnosis is hard to find, some features specific to the pathomimesis can be described. Indeed, cutaneous injuries are generally noted on accessible and contra lateral zones of the dominant limb with sometimes relatively geometric forms. ${ }^{[10]}$

If the wound's microbiological analysis shows a polymicrobial flora, we will suspect a hand manipulation of this wound. ${ }^{[11]}$ Foreign bodies can be found there and the histology will then reveal a reaction against foreign bodies.

Moreover, these patients are frequently depressed, subject to emotive reactions, often in conflict with family members and having problems with acquaintances. ${ }^{[12]}$ 
Finally, a simple test that facilitates the self-maiming proves that the lesions disappear under an occlusive dressing and reappear after its removal. This test is to be done under hospital control, creating a delay in the reappearance of the lesion and denying the patient any opportunity for self-mutilation.

\section{A thorough psychoanalytic aspect of Munchausen's} syndrome is out of this article's perspective. We deduce that the self-mutilation by these patients to produce some symptoms reveals a severe psychiatric disturbance, even though it is difficult to pinpoint. Usually, these patients are extremely reluctant to any psychological approach, even though it may be curative. When the diagnosis is made, the misled doctors are not always able to propose a necessary psychiatric follow-up. ${ }^{[5]}$ Indeed, to obtain the best results, this kind of follow-up may need to be accompanied by psychotropic drugs.

\section{CONCLUSION}

Despite recent articles regarding the treatment of factitious disorders, in actual situations, these diagnoses often remain ignored, with sometimes fatal consequences. Moreover, the economic consequences are often considerable. A factitious disorder must be taken into account every time manifest pathological symptoms are not accompanied by the signs usually associated with them. Once the proper diagnosis is made, the real work on the patient can start. It is our recommendation that to avoid continuing failure in the treatment of factitious diseases, there must be close collaboration between the 'somatic' specialist doctor, psychiatrist and general practitioner.

\section{ACKNOWLEDGMENTS}

The authors wish to thank Dr. K. Hadoun, psychiatrist of the Hopital Psychiatrique de Glain, Liege for his collaboration on certain psychological aspects of this article. In Memoriam: This article is dedicated to the memory of Dr. Olivier Heymans (1968-2007), a brilliant surgeon who inspired many of us.

\section{REFERENCES}

1. American Psychiatric Association. The diagnostic and statistical manual of mental disorders. $4^{\text {th }}$ ed. American Psychiatric Association: Washington; 1994. p 471-5.

2. Eisendrath SJ. Factitious physical disorders: Treatment without confrontation. Psychosomatics 1989;30:383-7.

3. Hamolsky MW. Truth is stranger than factitious. N Engl Med 1982;307:436-7.

4. Reich P, Gottfried LA. Factitious disorders in a teaching hospital. Ann Intern Med 1983;99:240-7.

5. Aduan RP, Fauci AS, Dale DC, Herzberg JH, Wolff SM. Factitious fever and self-induced infection: A report of 32 cases and review of the literature. Ann Intern Med 1979;90:230-42.

6. Folks DG, Freeman AM. Munchausen's syndrome and other factitious illness. Psychiatr Clin North Am 1985;8:263-78.

7. Masterton G. Factitious disorders and the surgeon. $\mathrm{Br} J$ Surg 1995;82:1588-9.

8. Paar GH. Factitious disorders in the field of surgery. Psychoter Psychosom 1995;62:41-7.

9. Mahler D, Ginath Y. The "Münchausen syndrome" in aesthetic surgery of the nose: The patient's problem or the surgeon's problem? Ann Plast Surg 1982;8:258-62.

10. Agris J, Simmons CW. Factitious (self-inflicted) skin wounds. Plast Reconstr Surg 1978;62:686-92.

11. Castor B, Ursing J, Aberg M, Pålsson N. Infected wounds and repeated septicemia in a case of factitious illness. Scand J Infect Dis 1990;22:227-32.

12. Basex A, Corraze J. Les pathomimies. Rev Prat 1966;16:2073-84.

Source of Support: Nil, Conflict of Interest: None declared. 